\title{
Determining the nature of prefrontal cortex recruitment after traumatic brain injury: a response toTurner
}

\author{
Frank G. Hillary* \\ Psychology Department, Pennsylvania State University, University Park, PA, USA \\ ${ }^{*}$ Correspondence: fhillary@psu.edu
}

\section{A commentary on}

Prefrontal compensatory engagement in TBI is due to altered functional engagement of existing networks and not functional reorganization

by Turner, G. R., McIntosh, A. R., and Levine, B. (2011). Front. Syst. Neurosci. 5:9. doi: 10.3389/fnsys.2011.00009

There remains uncertainty in the clinical neurosciences about how prefrontal cortex accommodates neurological insult and, in particular, the implications altered prefrontal responsivity has for recovery. In the functional imaging literature using blood oxygen level dependent functional magnetic resonance imaging (fMRI) methods, it has been consistently demonstrated that individuals with moderate and severe traumatic brain injury (TBI) "recruit" regions of prefrontal cortex. This literature has focused on fMRI tasks requiring rapid information processing and working memory (McAllister et al., 1999, 2001; Christodoulou et al., 2001; Maruishi et al., 2007; Newsome et al., 2007; Scheibel et al., 2007, 2009; Sanchez-Carrion et al., 2008a,b; Turner and Levine, 2008). One central question unanswered in this literature is whether increased prefrontal cortex involvement observed after brain injury, represents: (1) a transient and natural recruitment of existing resources or (2) functional "brain reorganization" resulting in permanent incorporation of resources not previously involved in the task. In the February issue of Frontiers in Systems Neuroscience, a paper by Turner et al. (2011) examines this question specifically.

While the authors offer a creative analytic approach in addressing this question, I have two concerns when reading their paper: the first has to do with defining and identifying "neural recruitment" to directly test brain reorganization hypotheses and the second has to do with the authors' explanation for what recruitment in prefrontal cortex represents. In the following, I will explain these concerns with the goal of exploring how we might determine the nature of prefrontal cortex recruitment after injury.

\section{IDENTIFYING PREFRONTAL RECRUITMENT}

In order to determine what prefrontal cortex is doing (or doing differently) after head trauma, several authors have emphasized examining the relationship between brain activation and task performance (Perlstein et al., 2004; Newsome et al., 2007; Hillary, 2008). Isolating this relationship permits identification of those neural systems that wax and wane in correspondence with changing task performance. This relationship is meaningful because when neural responsivity and performance are coupled, it is thought that altered brain activation represents natural fluctuations in the allocation of resources over the course of a task as opposed to more permanent reorganization of the system. Establishing the relationship between activation and performance is fundamental to the work by Turner et al. (2011). In fact, they go so far as to define "activation" in their study as the relationship between the fMRI signal and task accuracy; thus the fMRI signal is modeled solely as it relates to task accuracy. While this approach does link the fMRI signal to behavior, in doing so, the authors are also unlikely to observe all possible neural resources that are differentially recruited after injury. For example, there may be neural responses that hold non-linear relationships with accuracy or are related only to reaction time. Unfortunately, this limited definition of "activation" also influences interpretation of the authors' connectivity analysis which includes an original approach for examining covariance in task-related areas after TBI. One way to handle this issue is to conduct independent, but comparable analyses, that alternately include and exclude performance variables. To examine this question in TBI, we conducted whole brain analyses with and without reaction time as a regressor for the
fMRI signal, thus permitting two separate observations that can be compared. The result demonstrated overlap between those prefrontal regions that differentiated the groups (i.e., recruitment) and prefrontal regions that were positively correlated with reaction time (Hillary et al., 2010).

A related concern with the approach used in Turner et al. (2011) has to do with how they define and examine "brain reorganization." In order to directly test brain reorganization hypotheses, the authors contend that any prefrontal cortex involvement that is unique to the brain injured sample and is also related to task performance could be interpreted as "brain reorganization.” Turner et al. (2011) test this assumption by measuring the spatial overlap between TBI and healthy control groups. That is, if the groups share regions of prefrontal involvement, the observed effect may be deemed "inconsistent with the notion of reorganization whereby new network nodes not previously engaged by the task are related to task performance" (p. 8). The problem is that the authors have no way to identify prefrontal involvement after TBI that is not correlated with performance, an outcome that could be a candidate for brain reorganization. Moreover, the results from this approach may be misleading given that variance in accuracy data is artificially restricted in fMRI studies; investigators must guarantee high accuracy rates to ensure task compliance. With this method it is unclear that the authors have either identified neural recruitment as it has been observed in the literature or given themselves a chance to test competing hypotheses. The most important concern here is less tied to the relative success in refuting a brain reorganization hypothesis this hypothesis is difficult to definitively test with imaging methods and offers little new information about what prefrontal cortex is actually doing after injury. What is pivotal is that the authors' conclusion about how prefrontal cortex is responding to injury is 
based upon incomplete information thus limiting how these findings might inform us more generally about how prefrontal cortex accommodates injury.

\section{INTERPRETATION: THE MEANING OF ALTERED FUNCTIONAL ENGAGEMENT}

The authors conclude that after TBI, prefrontal engagement is compensatory and that this response does not represent functional brain reorganization. They also maintain that neural resources in prefrontal cortex recruited secondary to TBI serve very similar functions to those in healthy adults, but may be observable at lower task loads after injury. There is an intellectual history for this position and, on these points, we agree with the authors (see Hillary et al., 2006, 2010; Hillary, 2008). We anticipate that the recruitment of prefrontal cortex after injury represents the allocation of "latent resources" already in place and, as seen in healthy adults, the demand for these resources diminishes with increased task facility. In fact, our lab recently demonstrated that with task practice, right prefrontal cortex involvement diminishes almost identically in individuals with injury and their healthy control counterparts (see Medaglia et al., 2011).

While the innovation in the "altered functional engagement hypothesis" is not entirely clear, there is one important difference between our position and the interpretation offered by Turner et al. (2011). The authors argue that recruitment of resources after injury can be compensatory, but only under some conditions. For example, they conclude that neural recruitment at lower task loads may be compensatory, but that increased prefrontal involvement at higher task loads in TBI is attributable to "poor regulation of functional brain activity." This conclusion is puzzling and makes one wonder how neural recruitment after injury might simultaneously facilitate performance at one task load, yet be indicative of abnormal neural "regulation" at another. It is equally dubious that functional engagement of prefrontal areas differs at each task load yet activation at all loads remains correlated with the same performance variable. In the end, even if we accept that the approach taken in Turner et al. (2011) appropriately measures prefrontal recruitment and successfully refutes competing hypotheses, the interpretation of these data is difficult to decipher and our understanding of the nature of prefrontal recruitment after injury remains unchanged.

\section{SUMMARY: MULTIPLE ROLES FOR PREFRONTAL CORTEX AFTER INJURY}

The goal remains to determine what prefrontal recruitment represents after injury, and while I have been critical of this work by Turner et al. (2011), it is clear that gaining traction on this issue is not simple. When considering this literature broadly, part of the difficulty may be attributable to the potentially distinct response to injury occurring across functionally discrete nodes within prefrontal cortex. Ultimately, we anticipate that at least part of what has been observed as prefrontal recruitment in TBI represents the unmasking of latent resources during periods of inefficient task processing. This does not, however, preclude the possibility that subcomponents within prefrontal cortex operate to facilitate performance under certain conditions or at certain thresholds.

In this sense, the role of prefrontal cortex recruitment in working memory is multifaceted and there is unlikely to be uniform dedication of the same resources throughout the life of a task. For example, in studying TBI, reductions in right prefrontal connectivity can be observed from "early" to "late" in a task as learning occurs (see Hillary et al., 2011). Moreover, the relationship between activation and performance may depend upon how performance is measured. One might imagine a scenario where resources are necessary and sufficient for completion of a task (i.e., accuracy), but involvement of these same resources does not also guarantee rapid and efficient responding (i.e., reaction time). Thus, to determine the potentially divergent roles of prefrontal cortex recruitment on task performance after TBI, several nuanced manipulations will be required in combination. Future research should focus on within-subject and within-task changes in the neural and behavioral responses, connectivity analyses to examine prefrontal communication with other network regions (such as those offered in Turner et al., 2011 here), and longitudinal designs to observe the evolution of prefrontal involvement during critical windows of recovery.

\section{ACKNOWLEDGMENTS}

I thank Douglas L. Chute, Stephen T. Moelter, and Philip Schatz for their helpful comments in the preparation of this commentary.

\section{REFERENCES}

Christodoulou, C., DeLuca, J., Ricker, J. H., Madigan, N. K., Bly, B. M., Lange, G., Kalnin, A., Liu, W., Steffener, J., Diamond, B., and Ni, A. (2001). Functional magnetic resonance imaging of working memory impairment after traumatic brain injury. J. Neurol. Neurosurg. Psychiatr. 71, 161-168.

Hillary, F. G. (2008). Neuroimaging of working memory dysfunction and the dilemma with brain reorganization hypotheses. J. Int. Neuropsychol. Soc. 14, 526-534.

Hillary, F. G., Genova, H. M., Chiaravalloti, N. D., Rypma, B., and DeLuca, J. (2006). Prefrontal modulation of working memory performance in brain injury and disease. Hum. Brain Mapp. 27, 837-847.

Hillary, F. G., Genova, H. M., Medaglia, J. M., Fitzpatrick, N. M., Chiou, K. S., Wardecker, B. M., and DeLuca, J. (2010). Speed of information processing deficits in traumatic brain injury: is less brain more? Brain Imaging and Behav. 4, 141-154.

Hillary, F. G., Medaglia, J. M., Gates, K., Molenaar, P. M. C., Slocomb, J., Peechatka, A., and Good, D. C. (2011) Examining working memory task acquisition in a disrupted neural network. Brain 134.

Maruishi, M., Miyatani, M., Nakao, T., and Muranaka, H. (2007). Compensatory cortical activation during performance of an attention task by patients with diffuse axonal injury: a functional magnetic resonance imaging study. J. Neurol. Neurosurg. Psychiatr. 78, 168-173.

McAllister, T. W., Saykin, A. J., Flashman, L. A., Sparling, M. B., Johnson, S. C., Guerin, S. J., Mamourian A. C., Weaver, J. B., and Yanofsky, N. (1999). Brain activation during working memory 1 month after mild traumatic brain injury: a functional MRI study. Neurology 53, 1300-1308.

McAllister, T.W., Sparling, M. B., Flashman, L.A., Guerin, S. J., Mamourian, A. C., and Saykin, A. J. (2001). Differential working memory load effects after mild traumatic brain injury. Neuroimage 14, 1004-1012.

Medaglia, J. D., Chiou, K. S., Slocomb, J., Fitzpatrick, N. M., Wardecker, B. M., Ramanathan, D., Vesek, J., Good, D. C., and Hillary, F. G. (2011). The less BOLD, the wiser: support for latent resource hypothesis after neurotrauma. Hum. Brain Mapp. (in press).

Newsome, M. R., Scheibel, R. S., Steinberg, J. L., Troyanskaya, M., Sharma, R. G., Rauch, R. A., Lia, X., and Levin, H. S. (2007). Working memory brain activation following severe traumatic brain injury. Cortex 43, 95-111.

Perlstein, W. M., Cole, M. A., Demery, J. A., Seignourel, P. J., Dixit, N. K., Larson, M. J., and Briggs, R. W. (2004). Parametric manipulation of working memory load in traumatic brain injury: behavioral and neural correlates. J. Int. Neuropsychol. Soc. 10, 724-741.

Sanchez-Carrion, R., Fernandez-Espejo, D., Junque, C., Falcon, C., Bargallo, N., Roig, T., Bernabeu, M., Tormos, J.M., and Vendrell, P. (2008a). A longitudinal fMRI study of working memory in severe TBI patients with diffuse axonal injury. Neuroimage 43, 421-429.

Sanchez-Carrion, R., Gomez, P.V., Junque, C., FernandezEspejo, D., Falcon, C., Bargallo, N., Roig-Rovira, T., Enseñat-Cantallops, A., and Bernabeu, M. (2008b). 
Frontal hypoactivation on functional magnetic resonance imaging in working memory after severe diffuse traumatic brain injury. J. Neurotrauma 25, 479-494.

Scheibel, R. S., Newsome, M. R., Steinberg, J. L., Pearson, D.A., Rauch, R.A., Mao, H., Troyanskaya, M., Sharma, R. G., and Levin, H.S. (2007). Altered brain activation during cognitive control in patients with moderate to severe traumatic brain injury. Neurorehabil. Neural Repair 21, 36-45.

Scheibel, R. S., Newsome, M. R., Troyanskaya, M., Steinberg, J. L., Goldstein, F. C., Mao, H., and Levin, H. S. (2009). Effects of severity of traumatic brain injury and brain reserve on cognitive-control related brain activation. J. Neurotrauma 26, 1447-1461.

Turner, G. R., and Levine, B. (2008). Augmented neural activity during executive control processing following diffuse axonal injury. Neurology 71, 812-818.

Turner, G. R., McIntosh, A. R., and Levine, B. (2011). Prefrontal compensatory engagement in TBI is due to altered functional engagement of existing networks and not functional reorganization. Front. Syst. Neurosci. 5, 1-12. doi: 10.3389/fnsys.2011.00009
Received: 11 April 2011; accepted: 25 April 2011; published online: 10 May 2011.

Citation: Hillary FG (2011) Determining the nature of prefrontal cortex recruitment after traumatic brain injury: a response to Turner. Front. Syst. Neurosci. 5:24. doi: 10.3389/ fnsys.2011.00024

Copyright (C) 2011 Hillary. This is an open-access article subject to a non-exclusive license between the authors and Frontiers Media SA, which permits use, distribution and reproduction in other forums, provided the original authors and source are credited and other Frontiers conditions are complied with. 